\title{
Velocity field measurements of cavitating flows
}

\author{
A. L. Tassin, C.-Y. Li, S. L. Ceccio, L. P. Bernal
}

\begin{abstract}
A particle Image Velocimetry (PIV) system has been developed to study the microfluid mechanics of cavitating flows. Planar PIV was used to examine the non-cavitating flow in the thin boundary layer near a hydrofoil surface for the cases of a naturally developing boundary layer and a boundary layer stimulated to turbulence by roughness near the foil leading edge. PIV was also used to examine the flow near the surface of individual cavitation bubbles and incipient attached cavitation. A system was devised to create a single nucleus in the flow upstream of a hydrofoil, and planar PIV was used to study the flow around the resulting traveling cavitation bubble. Velocity vectors were determined close to the solid surfaces and the gas/liquid interfaces of the bubbles. Seeding of the flow with particles did not result in the addition of active cavitation nuclei.
\end{abstract}

\section{1}

\section{Introduction}

The use of Lagrangian particle tracers for quantitative flow visualization has undergone substantial development in the past several years (Adrian 1986a; Adrian 1991). Particle Imaging Velocimetry (PIV), as this technique is often called, provides the opportunity to non-intrusively visualize large regions of a flow field with substantial spatial and temporal resolution. These techniques are now being employed to study multiphase flows (see, for example, Liu and Adrian 1993; Sridhar and Katz 1994). This paper describes the application of these methods to the study of cavitating flows.

Cavitating flows are often difficult to visualize. The time scales associated with cavitating flows are quite small. For example, individual traveling cavitation bubbles exist for only a few milliseconds and will collapse within microseconds. The inception of attached cavitation occurs within milliseconds ( $\mathrm{Li}$

Received: 16 May 1994/Accepted: 7 August 1995

\section{A. L. Tassin, C.-Y. Li, S. L. Ceccio}

Department of Mechanical Engineering and Applied Mechanics, University of Michigan, Ann Arbor, M1 48109-2121, USA

P. Bernal

Department of Aerospace Engineering, University of Michigan, Ann Arbor, M1 48109-2121, USA

Correspondence to: S. L. Ceccio

The authors would like to thank Po-Wen Yu for his assistance in the photographic aspects of the PIV system. This work was sponsored by the Office of Naval Research under contract N00014-93-1029, Dr.

Edwin Rood, technical monitor. and Ceccio 1994). The inception and form of cavitation may be strongly influenced by the viscous flow near cavitating surfaces, and the length scale associated with the liquid boundary layer near a cavitating surface is usually much smaller than the length scales associated with the developed cavitation. Furthermore, cavitation inception is often a random process with individual cavitation nuclei moving into regions of low pressure.

Consequently, cavitation research has frequently resorted to high speed photography in order to understand the process of cavitation inception. Researchers have artificially produced the cavitation event to permit its study (Van der Meulen and Van Renesse 1989), or naturally occurring cavitation has been repeatedly observed in the hope of capturing a composite picture of the inception process (Ceccio and Brennen 1991). Simple photography, however, cannot provide information about the flow field near the cavity inception.

The new developments in PIV represent an opportunity to examine the fluid mechanics of cavitating flows in a more quantitative manner. Planar PIV has been used successfully to investigate the unsteady flow field surrounding laser-induced cavitation bubbles in a quiescent flow (Vogel and Lauterborn 1988). Hydrodynamic cavitation, however, occurs in flowing systems. To study hydrodynamic cavitation with PIV, several challenges must be overcome:

- The cavitating flow occurs near a surface, and the regions of cavitation are defined by irregular fluid-vapor interfaces. These surfaces will scatter the interrogating laser light sheet, and may interfere with the observation of the particle tracers or illuminate other particles that are not within the plane of interrogation.

- Cavitation processes are strongly influenced by the number and type of active cavitation nuclei present in the flow. Consequently, the tracer particles must not act as cavitation nuclei.

- It is generally impossible to predict exactly where and when a cavitation bubble will occur in a real flow. Thus, for a given interrogation volume in the flow, much time and effort will be wasted waiting for a bubble to be captured in the interrogation volume. Therefore, triggering a cavitation bubble at a known time and place would permit an efficient and detailed study of the phenomenon.

Presented here is a PIV system that has been designed to study hydrodynamic cavitation. The methods used to overcome the difficulties outlined above are discussed, and sample PIV images and results are provided. PIV techniques are used to 
reveal the unsteady processes associated with attached cavitation inception and the interaction of cavitation bubbles with the viscous flow near a surface.

2

\section{Experimental Setup}

The PIV experiment is composed of several parts:

- the Blow-Down Water Tunnel (BDW'T)

- the cavitation hydrofoil

- the nucleus production apparatus

- the PIV imaging apparatus

- the particle flow seeding

- the synchronization controller

- the PIV image processing system

\section{1}

\section{Blow-down water tunnel}

Figure 1 shows a schematic of the Blow-Down Water Tunnel (BDWT) of the Cavitation and Multiphase Flow Laboratory at the University of Michigan. It consists of two 400 gallon tanks connected to both vacuum and pressure lines. A square contraction of area ratio $4.4: 1$ connects the upper tank to the test section. The test section has a square cross-section with an interior side length of $76.2 \mathrm{~mm}$ and is approximately $260 \mathrm{~mm}$ long.

To create flow in the test section, water is first drawn into the upper tank using air pressure on the free surface of the water in the lower tank. The water is then allowed to settle. High speed flow is then created in the test section by applying pressure to the water free surface of the upper tank, and applying vacuum to the free surface of the lower tank. The maximum test section freestream velocity, $U_{0}$, is $30 \mathrm{~m} / \mathrm{s}$, and the test section freestream static pressure, $P_{0}$, can range from near vacuum to $690 \mathrm{kPa}$. In these experiments, the BDWT was operated to produce a free stream cavitation number of 0.28 with a free stream velocity of $15 \mathrm{~m} / \mathrm{s}$. Here the cavitation number, $\sigma$, is defined as $\left(P_{0}-P_{v}\right) / \frac{1}{2} \rho U_{0}^{2}$ where $P_{v}$ is the liquid vapor pressure and $\rho$ is the liquid density. Approximately 10 to $20 \mathrm{~s}$ of steady flow can be produced. Manual valves are used to coarsely control the operating regime of the tunnel. The operating conditions during blow-down are monitored with a computerized controller.

The natural nuclei content of test section flow is determined by the dissolved air content of the water and the amount of time elapsed between runs. By allowing the water to settle prior to the blow down, the free stream nuclei will rise to the free surface of the water. In this way, almost all the active nuclei can be removed from the free stream. This feature of the BDWT is essential for these experiments, since the occurrence of random traveling bubbles would interfere with the experiments. A single run, including time to settle the water, takes approximately ten minutes.

The test section is accessed by four removable windows. In these experiments, one aluminum window is used to mount the hydrofoil under study. A second Lucite window is equipped with a $50 \mathrm{~mm}$ diameter optical flat, mounted flush to the flow surface. This flat permits the entry of the laser light beam used to create a cavitation nucleus. A third aluminum window is equipped with a $75 \mathrm{~mm}$ diameter optical flat flush mounted

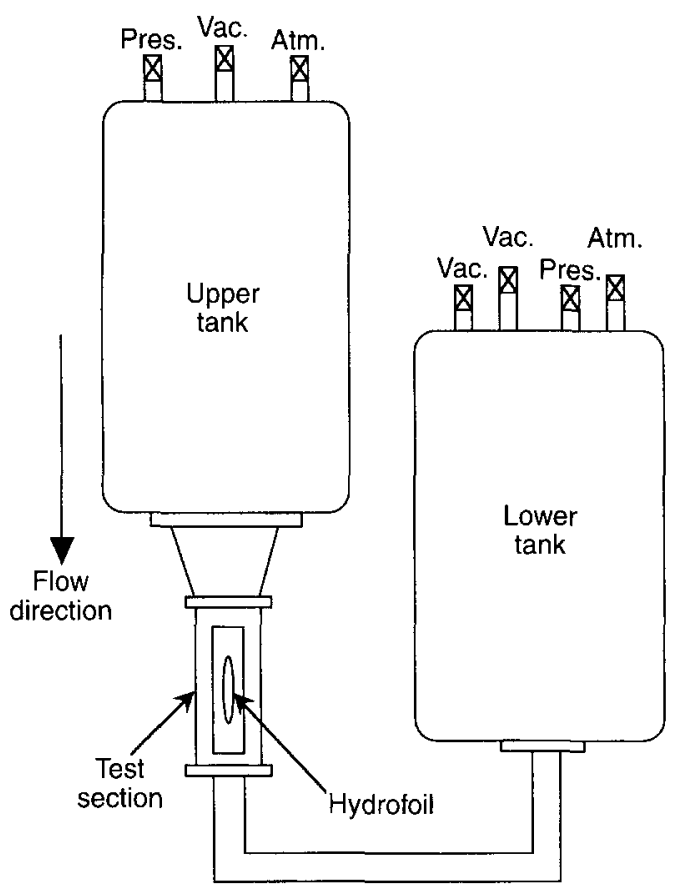

Fig. 1. Blow-down cavitation tunnel

with a flow to permit the entry of the laser light sheet necessary for the PIV. The last Lucite window permits observation of the flow illuminated by the PIV light sheet.

\section{2}

\section{Cavitation hydrofoil}

A two-dimensional hydrofoil with NACA section designation $63_{1}$ A012 was used in the experiments discussed below. The foil was made from free-machining brass and highly polished. It was mounted on one end in such a way as to permit variation in angle of attack from $-7^{\circ}$ to $+7^{\circ}$. The unmounted end of the hydrofoil was rounded to reduce the influence of side-wall cavitation on the view of the cavitating flow near the mid-span of the foil.

\section{3}

\section{Nucleus production apparatus}

Cavitation inception often results from the growth of a single cavitation nucleus. Nuclei are distributed throughout a flow making it impossible to know exactly when a single ambient nucleus will flow into the low pressure region. In order to study the micro-fluid mechanics of cavitation inception, it is advantageous to create a single nucleus at a known position in the flow. By creating the nucleus artificially, the location and timing of the incipient cavitation is made highly repeatable. Furthermore, by removing the naturally occurring active nuclei, randomly occurring cavitation events will not interfere with the flow of the artificially created event under examination. To permit such studies, a system was devised to create a single nucleus near the surface of the hydrofoil. Similar setups for creating laser-induced cavitation have also been used by other researchers (Lauterborn 1975; Lauterborn and Bolle 1975). 
The nucleus is created by vaporizing a small region of the flow near the hydrofoil leading edge with a focused laser light pulse. The experimental setup used to create the laser-induced cavitation nuclei is shown schematically in Fig. 2. A frequencydoubled Quantel 581C Nd-YAG laser is used to create the bubble. This laser can fire with a maximum repetition rate of $10 \mathrm{~Hz}$ and a maximum energy of $300 \mathrm{~mJ}$ in a 2 to $40 \mathrm{~ns}$ pulse. The pulse energy exiting the laser is approximately $140 \mathrm{~mJ} /$ pulse at a wavelength of $532 \mathrm{~nm}$. The original $7 \mathrm{~mm}$ diameter laser beam is expanded to a $42 \mathrm{~mm}$ diameter collimated beam using lens 1 (a meniscus lens, $-100 \mathrm{~mm}$ focal length) and lens 2 (a plano-convex lens, $600 \mathrm{~mm}$ focal length). Lens 3 (a plano-convex lens, $50 \mathrm{~mm}$ focal length, $25.4 \mathrm{~mm}$ diameter) is used to focus only a fraction of the collimated light beam into the test section. The rest of the beam is blocked by a beam stop. Lens 3 is on a three-axis traversing mechanism to facilitate the placement of the focal point in the test section. At the focal point of the laser beam, a cavitation nucleus is formed and swept up by the flow.

The nucleus produced with the above apparatus was on the order of $100 \mu \mathrm{m}$ and was quite repeatable in size, location and timing. Also, due to the asymptotic nature of cavitation bubble growth, any nucleus should grow to approximately the same size if it experiences the same pressure field as it flows over the hydrofoil (Ceccio and Brennen 1991). This was verified in the present experiment.

\section{4}

\section{PIV Imaging Apparatus}

Single-frame, double-pulsed, planar PIV is used to determine the velocity field in a plane near the incipient cavitation that resulted from the artificially produced nucleus. The plane of light is perpendicular to the surface of the hydrofoil, and parallel to the direction of the free stream flow.

Top and side views of the PIV optical setup are shown in Fig. 3. Two frequency-doubled Quanta Ray GCR130 Nd-YAG lasers are used to produce the double-pulsed light sheet. These lasers have a design repetition rate of $30 \mathrm{~Hz}$ with a maximum energy of $100 \mathrm{~mJ}$ in a $7 \mathrm{~ns}$ pulse. The two $7 \mathrm{~mm}$ diameter beams from the lasers are made collinear using a mirror and a beamsplitter. The beams are then brought to a line focus by lens 1 (a cylindrical lens, $60 \mathrm{~mm}$ focal length) and collimated by lens 2 (a cylindrical lens, $6.35 \mathrm{~mm}$ focal length). This yields a light sheet with a thickness of approximately $0.75 \mathrm{~mm}$ and a height of $7 \mathrm{~mm}$. Lens 3 (a plano-convex lens, $50 \mathrm{~mm}$ focal length) is used to increase the height of the light sheet.

Since the light sheet is composed of light from two in-line $\mathrm{Nd}$-YAG lasers, the delay between pulses can be greatly varied. The light scattered by tracer particles in the fluid is captured on a single negative by a $35 \mathrm{~mm}$ Nikon camera aimed in a normal direction to the light sheet. The camera is equipped with a Micro-Nikkor $\mathrm{f} 2.8 / 105 \mathrm{~mm}$ lens reversed to correct for aberrations. Two fully extended bellows are placed between the lens and camera. High resolution TMAX 400 film is used. The result is a magnified image of particle pairs in the flow field. The $35 \mathrm{~mm}$ negative is a 3.44 magnification of the real image.

One drawback of single-frame, double-pulsed PIV is the ambiguity in determining the flow direction, i.e., there is no way to know which particle came first in the pair without a priori information about the flow (Adrian 1986a, b). For the

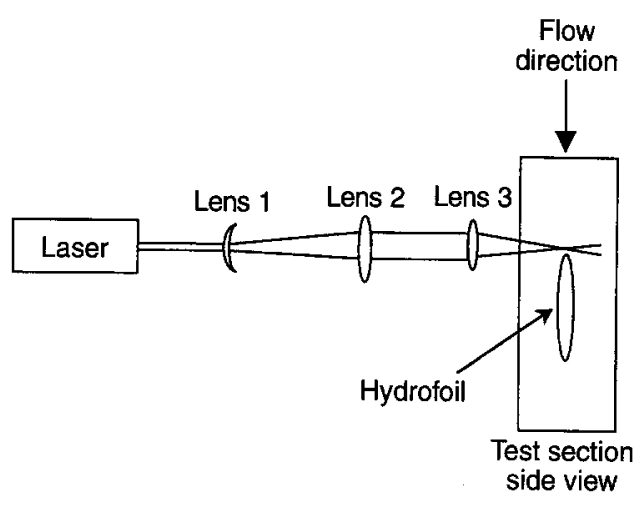

Fig. 2. Laser-induced nuclei experimental setup

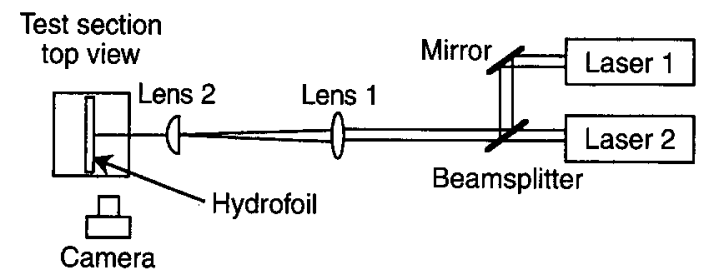

Top view

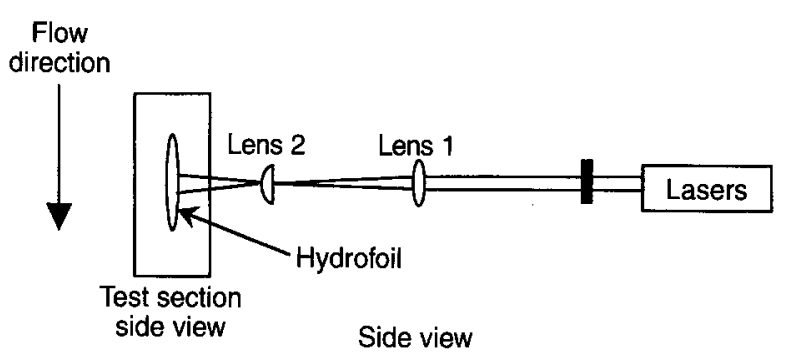

Fig. 3. PIV experimental setup

cases studied here, the flow is not expected to reverse, so this directional ambiguity will not affect the results. For studying separated flows, there are several methods for removing the directional ambiguity. One method would be to use singleframe, triple pulsed PIV by adding a third Nd-YAG laser to the double-pulsed set up. By having different time delays between the three pulses, it will be easy to determine the direction of the flow as long as the particle remains in the light sheet for all three pulses. Other methods of resolving the directional ambiguity would be to use a set up involving a rotating mirror or a drum camera (Adrian 1991).

\section{5}

\section{Particle flow seeding}

The flow is seeded with $\mathrm{TiO}_{2}$ particles of nominally $3 \mu \mathrm{m}$ diameter. The specific gravity of $\mathrm{TiO}_{2}$ is 4.2 . Over time, the particles would settle to the bottom of the BDWT. While the density of the particles does not exactly match that of the water, it is expected that the particles will track the flow due to their small size and the relatively small density difference (Durst et al. 1981). The time constant for a $3 \mu \mathrm{m}$ particle to respond to a step change in flow velocity is $2 \mu$ s based upon Stoke's drag of a sphere. This indicates that the particles should follow the flow with a $1 \%$ error or less for the flows under 
consideration here, based on the measured velocity gradients in the shear layer near the surface of the hydrofoil. The concentration of the particles in the water was varied from approximately $75000-150000$ particles $/ \mathrm{ml}$.

It was determined that flow seeding did not introduce significant numbers of active cavitation nuclei within the flow. Particles can act as nuclei when small amounts of gas on the particle surface grow in regions of low pressure. In this experiment, the particles were very small, making the cavitation inception number for these nuclei quite low. The particles were originally in a dry powder form; they were mixed with water and sonified to break up agglomerations and remove some of the gas trapped on the particle surfaces. To further reduce the cavitation number at which the particles would become active nuclei, the particles were placed in the de-aerated water of the BDWT for at least one day to permit any free gas on their surface to dissolve into the surrounding liquid. Thus, it was possible to heavily seed the BDWT with particles while simultaneously operating with very few active free stream nuclei.

\section{6}

\section{Synchronization controller}

Coordination of the laser-induced nucleus and the PIV system is necessary to ensure that the PIV image can capture the cavitating flow at any desired moment as well as control the duration between the PIV light pulses.

A single controller is used to coordinate all three lasers. The following sequence occurs during the capture of one PIV image. First, the "nuclei" laser is fired to produce a single nucleus. The nucleus will travel into the low pressure region of the flow near the foil surface and subsequently grow into a cavitation bubble. After a delay, the two PIV lasers are fired to capture the double-pulsed image of the incipient cavitation and the surrounding flow.

There are two main time delays. The first is the timing delay between the firing of the bubble-inducing laser and the firing of the first PIV laser. The second time delay is between the firing of the two PIV lasers. Once the time delays are set appropriately, each laser-induced bubble and the corresponding double-pulsed image can be reliably captured on film. Note that the Nd-YAG lasers cannot be fired asynchronously. The flash lamps, which charge the laser rods, must be continually fired at or near their design rate in order to maintain the operating temperature of the $\mathrm{Nd}-\mathrm{YAG}$ rods. The flash lamps must also be synchronized. In this experiment, synchronization was made difficult by the different flash rates of the three lasers $(10 \mathrm{~Hz}$ for the nuclei laser, and $30 \mathrm{~Hz}$ for the two PIV lasers). A master signal was used to drive the PIV lasers' flash lamps, and the nuclei laser flash lamps. The signals to the three $Q$-switches to fire the three lasers were also derived from the master signal, after appropriate time delays. The circuit also permits the optimization of the delay between the flash lamps and the $Q$-switch of each laser to maximize the pulse energy. The controller can operate in single shot or continuous firing modes.

\section{7}

\section{PIV Image Processing}

The PIV imaging system produces a double-pulsed image of the incipient cavitation and the tracer particles in the sur- rounding flow. By analyzing the displacement of the particles, the local velocity can be inferred.

The method of processing the PIV images for this study involves the direct measurement of the displacement between each particle pair. The PIV negative is first enlarged and printed. This step increases the overall magnification another 12.5 times to 43.0 . The print is then scanned using a high resolution scanner at $600 \mathrm{dpi}$ (optical). This digital image is analyzed on a Macintosh Power PC 6100/60 40/250 computer using the public domain program NIH Image 1.57 (Rasband 1995). With this program, the position of each particle is determined by an algorithm that calculates the center of the best fitting ellipse for each particle. From the particle positions and the time delay between exposures, the velocity vector of the particle is calculated.

Since NIH Image uses an algorithm to find the center of the best fitting ellipse, the only analysis parameters which can vary are the greyscale threshold level and the choice of the particle pairs. If two users set the same threshold level for an image and choose the same particle pair, then their results will be identical. By using forty randomly chosen particles from the PIV image shown in Fig. 4 and measuring the particle center at twelve different threshold levels from 30 to 140 , it was determined that the systematic error associated with thresholding in finding the particle center was approximately \pm 0.2 pixels. For these forty particles, the average particle area was 235.8 square pixels with an average major axis of 18.0 pixels and average minor axis of 15.8 pixels. For example, for all particle pairs found in Fig. 4, the magnitude of the position change between the particle pairs ranged from 25.0 to 51.6 pixels for
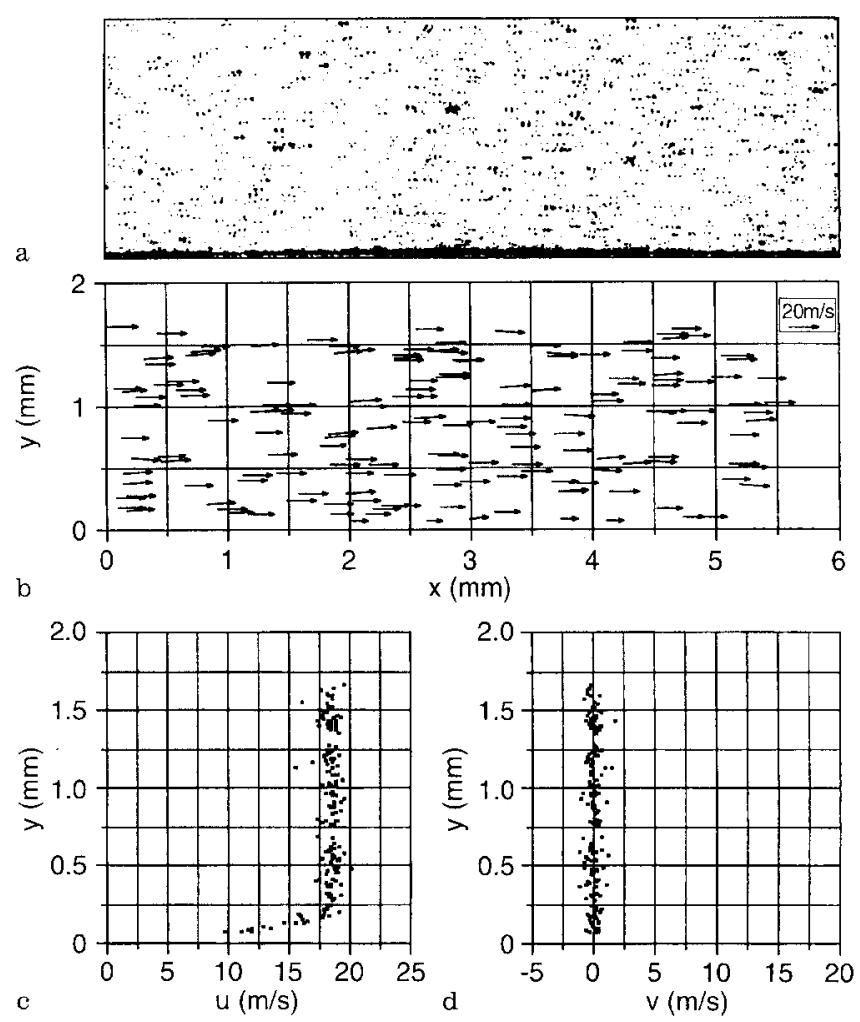

Fig. 4a-d. PIV analysis of naturally developing boundary layer flow. a double-pulsed image; b vector plot; $\boldsymbol{c}$ streamwise velocity $u$; d transverse velocity, $v$ 
the given $2.5 \mu$ s time delay. This corresponds to velocities of $9.8 \pm 0.2 \mathrm{~m} / \mathrm{s}$ to $20.3 \pm 0.2 \mathrm{~m} / \mathrm{s}$.

\section{3}

\section{Results}

The PIV system described above has been used to study local velocities within the fully wetted boundary layer over a hydrofoil for a natural developing boundary layer and for a boundary layer that had been artificially tripped. The interaction of a traveling cavitation bubble with the boundary layer near the cavitating surface was also examined.

In Fig. 4, a PIV image of the non-cavitating boundary layer flow over a hydrofoil is presented along with the analyzed velocity vectors. The flow is from left to right. It is possible to find particle pairs that lie inside the boundary layer, which is within $200 \mu \mathrm{m}$ of the hydrofoil surface. Each image typically yields 10 to 20 boundary layer vectors. The boundary layer in Fig. 4 is expected to be in a state of natural transition, and this is confirmed by the near surface pairs. These near surface pairs are parallel to the surface, although their streamwise $(u)$ velocity is reduced since they are located closer to the surface. This result is in contrast to the image in Fig. 5. In this case, the leading edge of the foil has been roughened thus creating a tripped turbulent boundary layer. As expected, the majority of the reconstructed velocity vectors near the surface of the hydrofoil are not as parallel to the surface. This can be seen in a comparison of vector plots as well as in the data spread near the surface in the transverse velocity $(v)$ plot. It can also be seen that the boundary layer in Fig. 5 is about $500 \mu \mathrm{m}$ thicker than the boundary layer in Fig. 4.
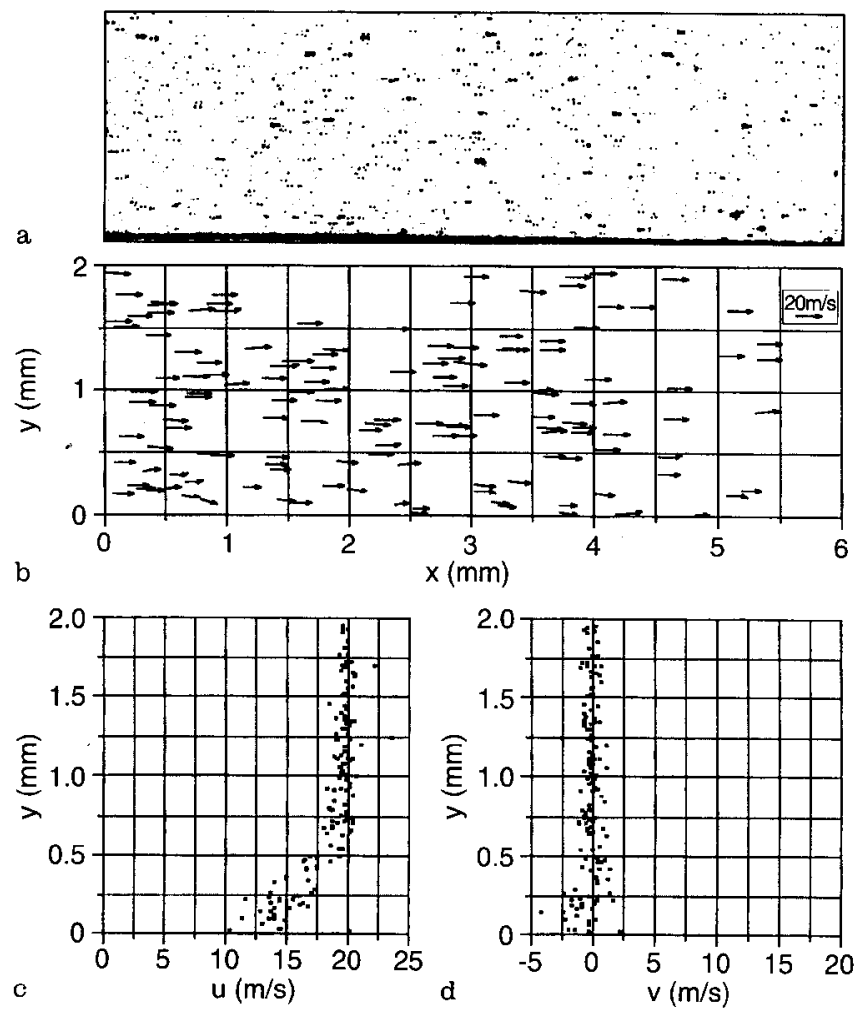

Fig. 5a-d. PIV analysis of tripped turbulent boundary layer flow. a double-pulsed image; $\mathrm{b}$ vector plot; $\mathrm{c}$ streamwise velocity, $u$; d transverse velocity, $v$
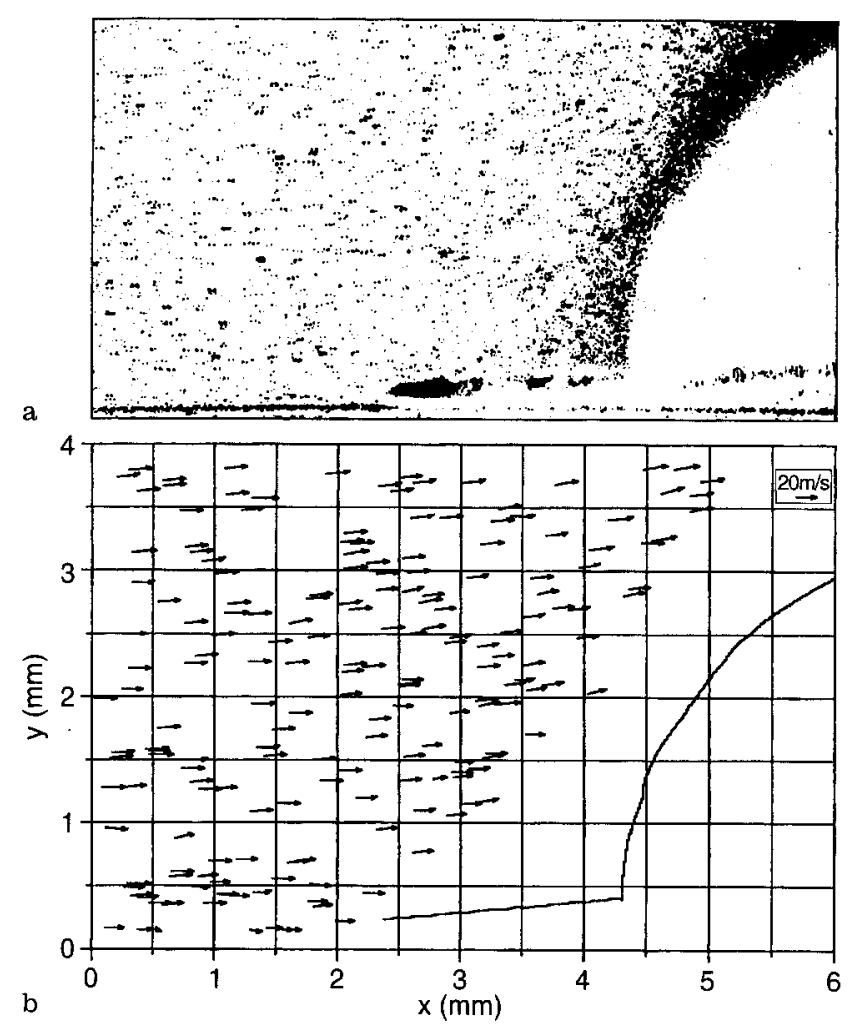

Fig. 6a, b. PIV analysis of flow behind a cavitation bubble. a double-pulsed image; $b$ vector plot with the outline of the bubble

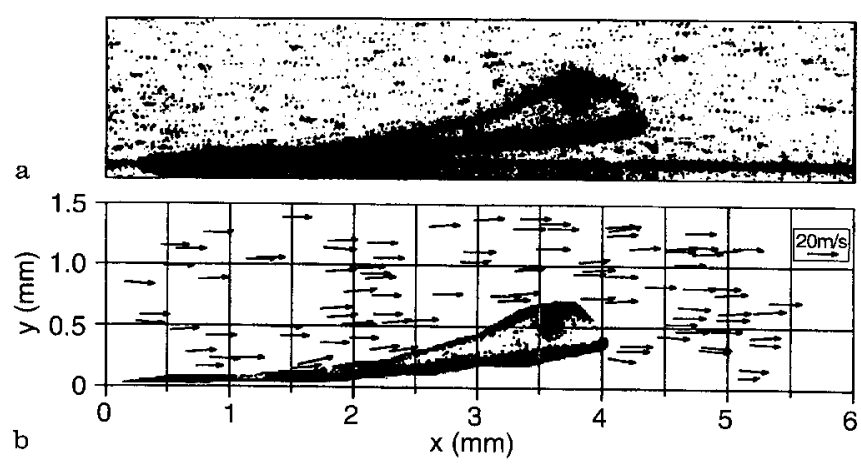

Fig. 7. PIV analysis of flow around surface cavitation. a doublepulsed image; $\mathbf{b}$ vector plot with the outline of the bubble

The PIV system can also be used to image the flow around cavitation bubbles. Figure 6 shows a travelling cavitation bubble that resulted from a laser-induced nucleus. Particle pairs are visible relatively near the bubble surface and upstream of the bubble. Around the bubble is an area of scattered light that makes it difficult to accurately determine particle pairs within one millimeter of the bubble's surface. Figure 7 shows an incipient surface cavity induced after the passing of a laser-induced traveling bubble. The flow around this cavity can also be visualized with PIV.

\section{4}

\section{Discussion and conclusions}

With the apparatus described in this paper, it is possible to produce planar PIV images of hydrodynamic cavitation. The 
following issues have been resolved:

- Double-pulsed planar PIV can be used to image hydrodynamic cavitating flows.

- The boundary layer may be visualized using PIV. Ensemble averaging may be used to better quantify the nature of the boundary layer.

- Cavitating flows can be seeded for PIV. Seed particles can be chosen that do not become active cavitation nuclei.

- It is possible to image particle pairs relatively near the surface of incipient and developed hydrodynamic cavitation. With the demonstrated ability to create individual cavitation bubbles on demand and to capture the resulting flow using PIV, it is possible to study the micro-fluid mechanics of cavitation inception, and bubble/boundary layer interactions.

\section{References}

Adrian RJ (1991) Particle-imaging techniques for experimental fluid mechanics: Ann Rev Fluid Mech 23: 261-304

Adrian RJ (1986) Multi-point optical measurements of simultaneous vectors unsteady flow-a review: Int J Heat Fluid Flow 7: 127-145

Adrian RJ (1986) Image shifting technique to resolve directional ambiguity in double-pulsed velocimetry. Appl Opt 25: 3855-3858
Ceccio SL; Brennen CF (1991) Observations of the Dynamics and Acoustics of Traveling Bubble Cavitation. J Fluid Mech 233: 633-660

Durst F; Melling A; Whitelaw JH (1981) Principles and practice of Laser-Doppler anemometry, 2nd ed., New York: Academic Press

Lauterborn W' (1975) Cavitation bubble dynamics-new tools for an intricate problem. Appl Sci Res 38: 165-178

Lauterborn W; Bolle H (1975) Experimental investigations of cavitation-bubble collapse in the neighbourhood of a solid boundary. J Fluid Mech 72: 391-399

Li CY; Ceccio SL (1994) Observations of the influence of nuclei content on the inception and form of attached cavitation. 20th Symp. on Naval Hydrodynamics, Santa Barbara

Liu ZC; Adrian RJ (1993) Simultaneous imaging of the velocity fields of two phases: Particulate two-phase flows, Ch. 2, Ed. M.C. Roco: Butterworth-Heinemann

Sridhar G; Katz J (1994) The effect of bubble clouds on vortex rings, ASME Cavitation and Multiphase flow forum, Lake Taho

Van der Meulen JHJ; Van Renesse RL (1989) The collapse of bubbles in a flow near a boundary: 17th Symp. on Naval Hydrodynamics, The Netherlands

Vogel A; Lauterborn W (1988) Time-resolved particle image velocimetry used in the investigation on cavitation bubble dynamics. Appl Opt 27: 1869-1876

Rasband W (1995) NIH image 1.57, U.S. National Institutes of Health, available by anonymous ftp from zippy.nimh.nih.gov or on floppy disk from NTIS, 5285 Port Royal Rd., Springfield, VA 22161, part number PB93-504868 